\title{
Advanced Usage of Vehicle Sketch Pad for CFD-Based Conceptual Design
}

\author{
Irian Ordaz ${ }^{1}$ and $\mathrm{Wu} \mathrm{Li}^{2}$ \\ NASA Langley Research Center, Hampton, VA, 23681
}

\begin{abstract}
Conceptual design is the most fluid phase of aircraft design. It is important to be able to perform large scale design space exploration of candidate concepts that can achieve the design intent to avoid more costly configuration changes in later stages of design. This also means that conceptual design is highly dependent on the disciplinary analysis tools to capture the underlying physics accurately. The required level of analysis fidelity can vary greatly depending on the application. Vehicle Sketch Pad (VSP) allows the designer to easily construct aircraft concepts and make changes as the design matures. More recent development efforts have enabled VSP to bridge the gap to high-fidelity analysis disciplines such as computational fluid dynamics and structural modeling for finite element analysis. This paper focuses on the current state-of-the-art geometry modeling for the automated process of analysis and design of low-boom supersonic concepts using VSP and several capability-enhancing design tools.
\end{abstract}

\section{Nomenclature}

$\begin{array}{ll}\text { AST } & =\text { Analytical Shaping Tool (a computer code for shape parameterization and deformation) } \\ \text { BOSS } & =\text { Boom Optimization using Smoothest Shape modification (a computer code for low-boom design) } \\ \text { CFD } & =\text { computational fluid dynamics } \\ \text { DOE } & =\text { design of experiments } \\ \text { FEA } & =\text { finite element analysis } \\ \text { PLdB } & =\text { perceived loudness in decibels } \\ \text { VSP } & =\text { Vehicle Sketch Pad } \\ \text { XML } & =\text { Extensible Markup Language } \\ A_{\mathrm{e}} & =\text { equivalent area } \\ A_{\mathrm{e}, \mathrm{r}} & =\text { reversed equivalent area } \\ \gamma & =\text { ratio of specific heat } \\ P & =\text { dimensional pressure } \\ P^{*} & =\text { non-dimensional pressure } \\ P_{\infty} & =\text { free-stream pressure } \\ X_{\mathrm{e}} & =\text { equivalent length }\end{array}$

\section{Introduction}

$\mathrm{Co}_{\mathrm{o}}$ onceptual design is the most fluid phase of the aircraft design cycle. At this early stage, the design is still evolving rapidly and the designer has significantly more design freedom than in later stages. Consequently, large scale design space exploration is typically performed to obtain the best configuration to meet the design requirements. Vehicle Sketch Pad (VSP) [1] is the preferred geometry modeling tool at this stage because it allows the designer to easily construct a configuration and make modifications as the design matures. The disciplinary analysis conducted during the conceptual design process is used to assess the viability, performance and economic merits of the design. The level of disciplinary analysis fidelity is dependent on the requirements to properly capture the underlying physics of the application. The early infusion of high-fidelity analysis is necessary to avoid more costly design changes in the later phases of design. VSP continues development to facilitate the automation of high-

\footnotetext{
${ }^{1}$ Research Engineer, Aeronautics Systems Analysis Branch, Systems Analysis and Concepts Directorate.

${ }^{2}$ Senior Research Engineer, Aeronautics Systems Analysis Branch, Systems Analysis and Concepts Directorate.
} 
fidelity disciplinary analyses such as computational fluid dynamics (CFD) and finite element analysis (FEA). This paper focuses on the CFD-based design capabilities using VSP and the capability-enhancing design tools that have been developed to enable the conceptual design of supersonic aircraft with low-boom and low-drag characteristics.

\section{Geometry Modeling Process}

The diagram shown in Fig. 1 depicts the data flow between VSP, the four capability-enhancing design tools, and the CART3D [2] CFD package. There are currently two main data flow paths that are used with the geometry modeling process starting with VSP. The first path uses the component-based point definition. Tools like SUPIN2HRM, BOSS, and PROTEUS operate on the component-based point definition of a VSP geometry which is typically written in the Hermite (structured grid) format. The second path uses the native parametric definition of VSP. Tools like AST operate on the native parametric definition of a VSP geometry by modifying the geometry parameters defined in the extended markup language (XML) model with shape functions.

Additional tools such as HRM2CART3D automate the process of refinement and patching of aircraft components into solid components, which can be triangulated and intersected with the CART3D CFD package to produce a CFD surface mesh. HRM2CART3D is also capable of trimming specific component intersections. An example is the trim of a pylon section which protrudes into the interior of a flow-through nacelle. This automation process enables automated numerical optimization using CFD analysis. GEO2VSP [3] is a tool capable of reconstructing a parametric VSP geometry model from the component-based point definition (e.g., Hermite or Plot3D). Thus, any configuration defined as a component-based point definition and modified with the capabilityenhancing design tools (SUPIN2HRM, BOSS, PROTEUS) can be re-parameterized in VSP format, and can be modified by using VSP. The focus of the paper is the group of tools at the top of Fig. 1 labeled SUPIN2HRM, BOSS [4], PROTEUS [5], and AST [6], as well as their applications. These tools have been developed to enhance the geometry modeling capability of VSP for design and analysis of low-boom and low-drag supersonic aircraft concepts.

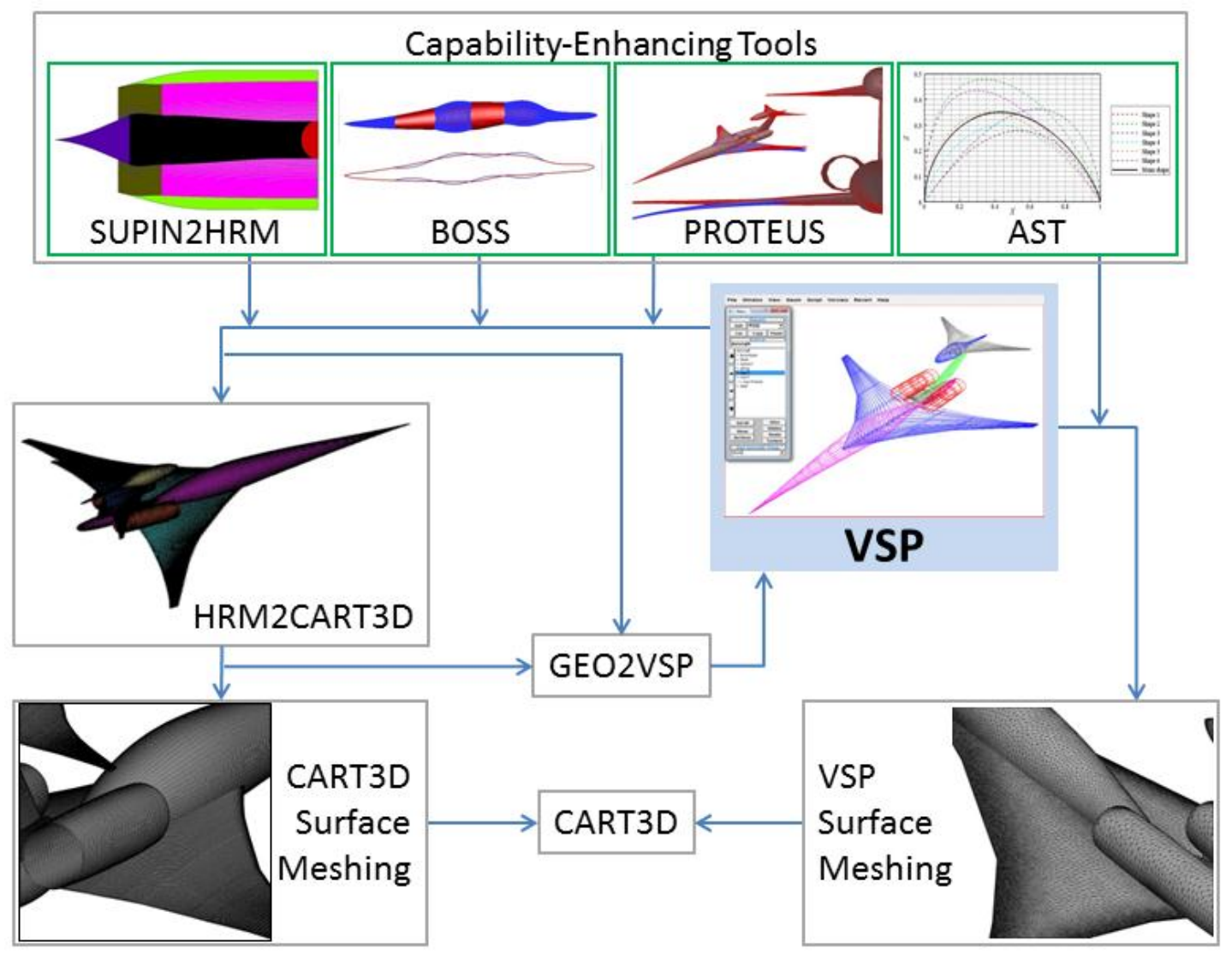

Figure 1. Geometry modeling process for CFD-based conceptual design. 


\section{A. Analytical Shaping Tool}

The Analytical Shaping Tool (AST) is a shape function-based geometry definition computer code. A shape function is a mathematical model that allows interpolation at locations where there are no geometry point definitions. The shape function implemented in AST was introduced by Kulfan and Bussoletti [7] and it is capable of describing fuselage radius and camber distributions, as well as lifting surface leading edge sweep, dihedral, twist, chord length, and airfoil thickness distributions. Furthermore, AST allows the definition of smooth spanwise distributions for parameters such as leading edge sweep and chord length which is not possible with VSP.

A low number of shape functions leads to a more rigid geometry, which reduces the feasible design space in which the optimizer can search for an optimal solution. Conversely, a larger number of shape functions leads to a greater number of design variables for the optimizer, which increases the computational effort and the time requirements. The shape function is a powerful concept which allows geometrical flexibility with varying degrees of freedom by simply varying the number of shape functions which describe the geometry.

\section{B. BOSS}

BOSS is a volume shaping tool used to modify non-lifting components for the design of low-boom supersonic aircraft. The designer can identify the discrepancy between the equivalent area of the configuration and a low-boom target to determine the region of the aircraft that needs to be modified to match the target. The design region can be input into BOSS by specifying the aircraft component (e.g., fuselage) and the corresponding range of equivalent length. BOSS allows volume shaping with over 120 design variables which greatly expands the design space for optimization. The result of BOSS shaping is either a low-boom configuration matching the equivalent area target or the elimination of any configuration infeasible for low-boom design with volume shaping alone [4]. This eliminates an open-ended trial-and-error process to determine the feasibility of a configuration for low-boom shaping.

\section{PROTEUS}

PROTEUS is a rapid shape parameterization tool for aircraft shape optimization. The tool can be used to deform geometries defined as component-based point definitions (as defined by the VSP Hermite format). Here, component-based point definition means that each aircraft component (e.g., wing) is defined as a single structured grid. Currently, there are eight parametric schemes which allow modifications of lifting and non-lifting components. Deformation of lifting components (e.g., wing and horizontal tail) includes planform, twist, thickness, camber, and localized surface changes. Deformation of non-lifting components (e.g., fuselage and pod) includes camber, height, width, and localized surface changes. In addition, it is possible to translate, rotate, and scale each component. The schemes allow entire or partial deformation along the spanwise and chordwise direction based on non-dimensional parameters ranging in value from 0 (root airfoil or airfoil leading edge) to 1 (tip airfoil or airfoil trailing edge). PROTEUS simplifies the implementation of parametric schemes which allows the designer to focus on the more important work of identifying the appropriate parametric scheme for the problem. It allows the sequential application of different parametric schemes on a baseline geometry. This feature is extremely useful in the search of a desired configuration by numerical shape optimization because of the difficulty associated with determining a parametric scheme a priori that will include a desired optimal shape.

\section{SUPIN2HRM}

SUPIN2HRM is a tool that can integrate a supersonic inlet generated by SUPIN [8] into a component-based point definition geometry generated by VSP. SUPIN is a conceptual design tool developed by John Slater at NASA Glenn Research Center for the analysis and design of external compression supersonic inlets based on analytical, empirical, and numerical low-fidelity methods. SUPIN2HRM reads the nacelle outer surface from VSP and blends it with the front part of the nacelle outer surface generated by SUPIN. The tool then combines all the surfaces generated by SUPIN to generate one structured mesh for the inlet, one structured mesh for the nacelle outer surface, and an optional structured mesh for an inlet spike. This approach allows automatic construction of the watertight engine model installed on an aircraft configuration for CART3D powered engine simulation. The most complex engine model generated by the automation process includes the inlet, spike, nozzle, nozzle plug, and aft bypass.

\section{Application of Capability-Enhancing Design Tools}

This section contains several case studies which demonstrate the application of four capability-enhancing design tools for design and analysis of low-boom and low-drag supersonic aircraft. These case studies have been previously published with the exception of the conceptual design and integration of a supersonic inlet with SUPIN and SUPIN2HRM. 


\section{A. Wing Planform Optimization with Analytical Shaping Tool}

A wing planform optimization performed with AST was demonstrated by Ordaz and Rallabhandi [6]. The goal of this study was to demonstrate an automated process for low-boom and low-drag optimization for supersonic aircraft using AST as the shape parameterization tool for the VSP geometry, and VSP as the surface meshing tool for automated CFD analysis. The flight conditions for the CART3D CFD analysis were cruise Mach number of 1.8, a cruise altitude of 51,650 ft, and a cruise weight of 45,000 lbs.

AST was used to vary the wing chord, sweep, and $x$-location with respect to the fuselage. A total of 26 design variables were used, including 12 shape function coefficients for the wing chord and sweep, respectively. Wing chord and sweep distributions each consisted of six shape functions including piecewise linear and nonlinear segments. The optimization objective was to reduce the range (i.e., the difference of maximum and minimum pressures) of the ground signature. The ground signature was calculated by propagating the equivalent area computed by the integration of volume and lift at the Mach plane cuts.

The optimum wing planform and non-dimensional pressure $\left(P^{*}=P / P_{\infty} \gamma\right)$ contours are compared to that of the baseline configuration in Fig. 2. The trailing edge of the optimized wing shows a greater nonlinear behavior because the increase in the number of shape functions has introduced more nonlinear degrees of freedom. The equivalent area comparison provided in Fig. 3 shows that the volume and lift contributions of the wing have shifted aft, thus reducing the overshoot of the equivalent area observed in the baseline configuration at $X_{e}=145 \mathrm{ft}$. The ground signature is propagated with ARAP [9]. The comparison of the ground signatures for the baseline and optimized configuration provided in Fig. 4 shows a decrease in the range of 16 percent. The optimized wing planform also resulted in a configuration with 10.5 percent increase in lift-to-drag ratio, and 3.7 percent decrease in PLdB.

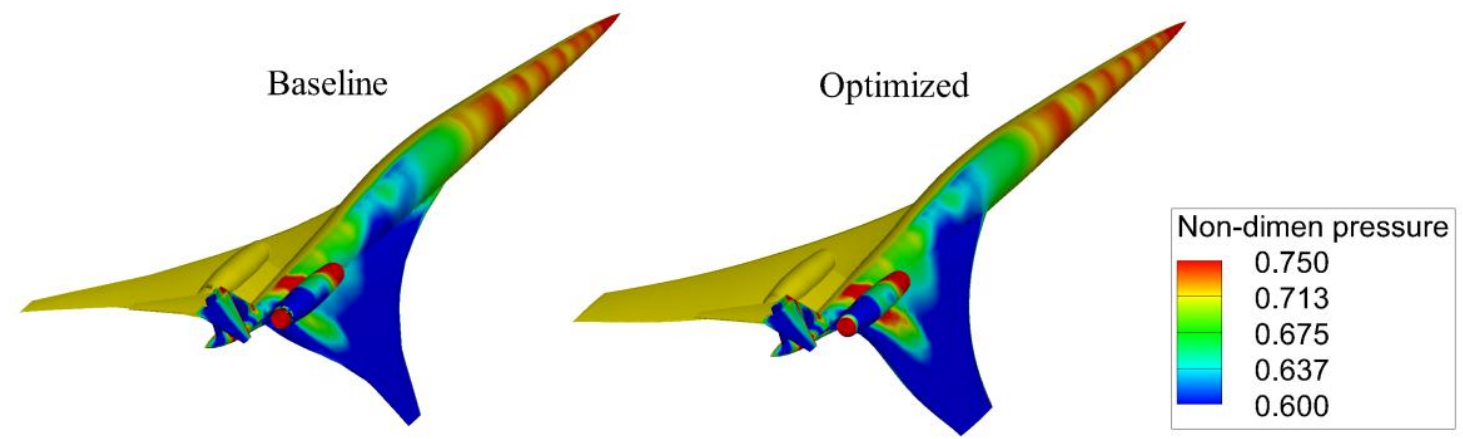

Figure 2. Comparison of CFD surface pressure contours for wing planform optimization with AST.

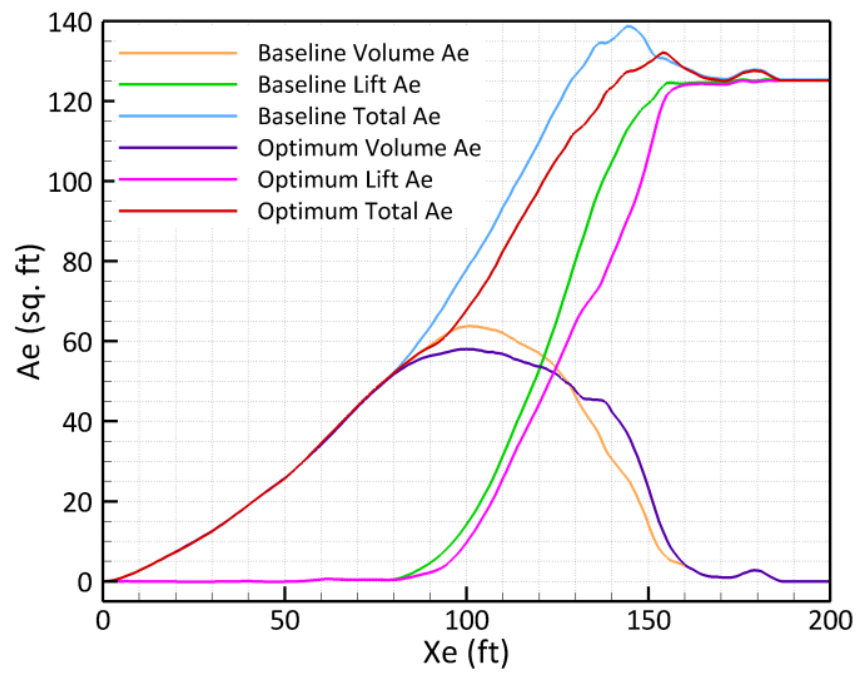

Figure 3. Comparison of equivalent area for wing planform optimization with AST.

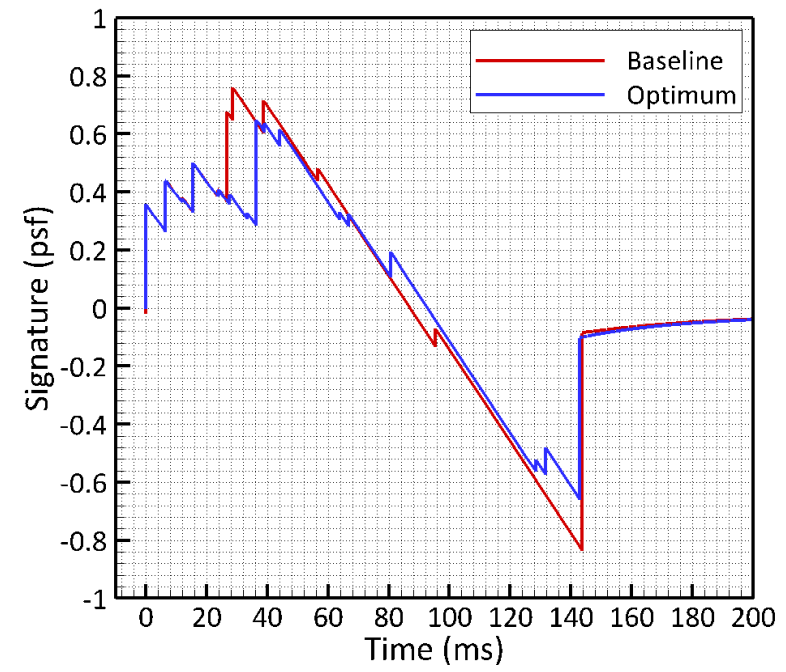

Figure 4. Comparison of ground signature for wing planform optimization with AST. 


\section{B. Equivalent Area Target Matching with BOSS}

A case study for volume shaping with BOSS was demonstrated by Li and Rallabhandi [10] to shape the ground signature propagated from an off-body pressure distribution. The design process consists of fuselage and pod shaping for matching reversed equivalent area $\left(A_{\mathrm{e}, \mathrm{r}}\right)$ to a low-boom target, followed by a design of experiments (DOE) for modification of the aft shape of the reversed equivalent area. The reversed equivalent area is computed by propagating an off-body pressure distribution backward in time using the Burgers' equation [11] and converting to the area of an equivalent body of revolution. The baseline configuration was originally designed by using a mixedfidelity $A_{\mathrm{e}}$ matching process [4] for a cruise weight of 30,000 lbs, a cruise Mach number of 1.6, and a cruise altitude of 45,000 ft. The baseline configuration is shown in Fig. 7.

Once the difference between the target equivalent area and the reversed equivalent area is relatively small in comparison to the fuselage equivalent area, one can scale the area distribution of the fuselage/pod cross sections to eliminate the difference and obtain a low-boom configuration [10]. The difficulty for the fuselage volume shaping is the determination of a parameterization scheme for the fuselage area distribution that will yield the required distribution for a low-boom configuration. Instead of using a trial-and-error method, an effective method of using smoothest shape modifications (called BOSS) with a high number (e.g., over 120) of design variables can be used to obtain the least oscillatory fuselage shape that yields a low-boom configuration [6,12].

Figures 5 and 6 show the reversed equivalent area distributions and the corresponding ground signatures for the baseline and the final design. The ground signatures are propagated with SBOOM [11] from $d p / p$ at three body lengths below the configuration. BOSS volume shaping, along with a horizontal tail DOE, was able to tailor the aft ground signature with a reduction of approximately $4 \mathrm{PLdB}$ in the ground signature. The cruise weight for the optimized configuration decreased to $27,000 \mathrm{lbs}$ as a result of the modifications to the horizontal tail. See [10] for more details.

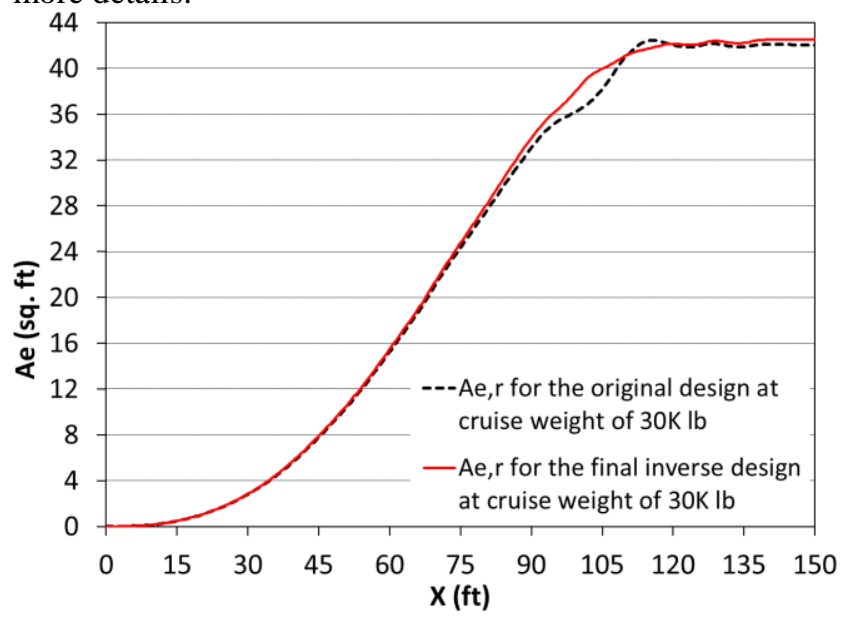

Figure 5. Reversed equivalent area for original and final designs.

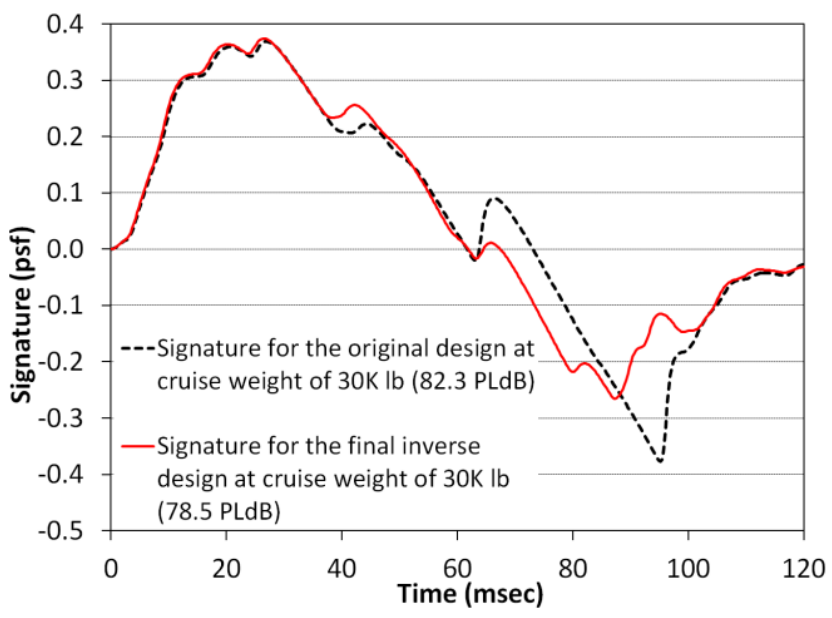

Figure 6. Ground signatures for original and final designs.

\section{Horizontal Tail Lift Tailoring with PROTEUS}

A horizontal tail lift tailoring optimization was demonstrated with PROTEUS by Ordaz and Li [12] for the configuration shown in Fig. 7. The baseline configuration was the result of the previous equivalent area target matching conducted with BOSS. The angle of attack was increased from 0.5 deg to 0.8 deg to achieve a desired cruise weight of 33,000 lbs. The cruise Mach number and altitude remain 1.6 and 45,000 ft, respectively. PROTEUS was used to conduct this aft lift optimization because it allows greater control of airfoil shape deformation beyond what is capable with VSP.

An automated optimization process was used to drive the reduction in the variation of the ground pressure signature from a linear target in the aft portion of the signature. The linear target was computed at each optimization iteration through a least-squares fitting of the pressure in the aft portion of the ground signature. This optimization objective seeks to reduce the pressure oscillations around the target.

The parametric scheme used for this horizontal tail optimization is shown in Fig. 8. The scheme consists of eight total design variables. Seven of these design variables (Var1 through Var7) control the camber at three airfoil locations. Three camber control points were placed at the leading edge, midchord, and trailing edge of the root and midspan airfoil locations. Another camber control point was placed at the trailing edge of the tip airfoil. Lastly, the 
spanwise location (Var8) of the midspan airfoil section was allowed to vary. Figure 9 shows a comparison of the baseline and optimum ground signatures propagated with SBOOM, including the desired reduction in large pressure fluctuations in the aft region and a loudness reduction of 3.8 PLdB. The comparison of the baseline and optimized horizontal tail geometries given in Fig. 10 shows an increase in twist at the root.

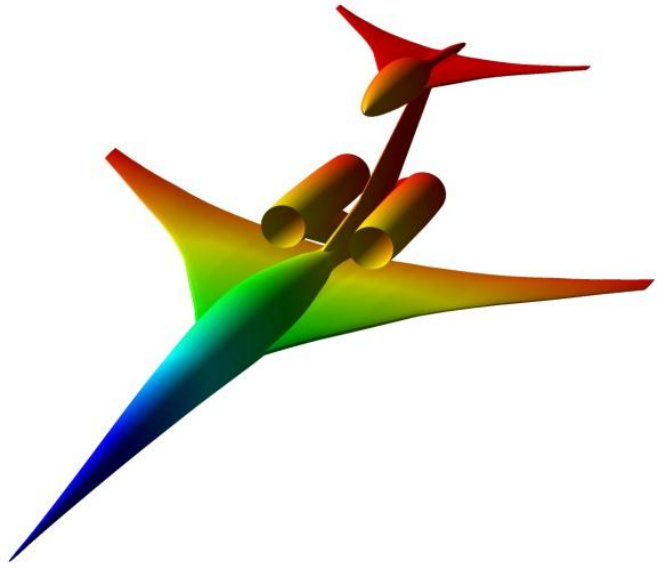

Figure 7. Baseline configuration for horizontal tail lift tailoring with PROTEUS.

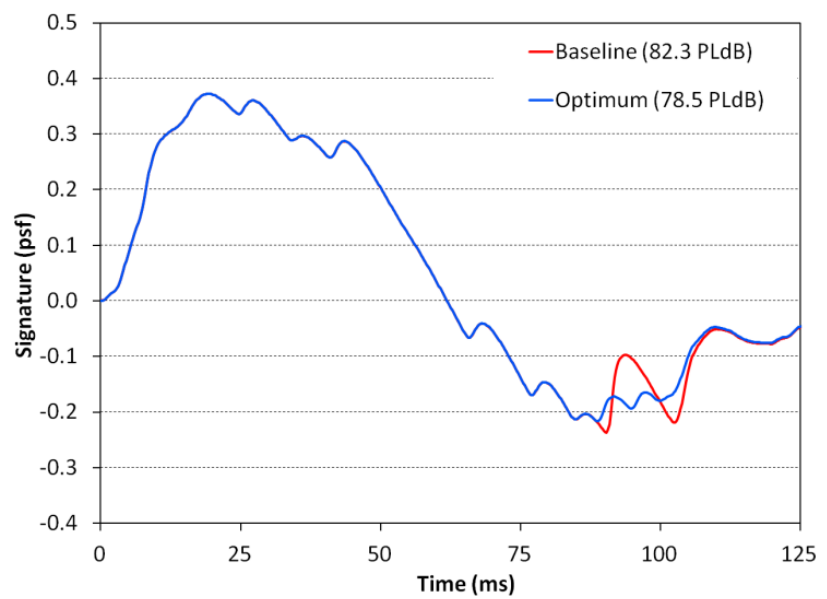

Figure 9. Comparison of baseline and optimized ground signatures for horizontal tail lift tailoring with PROTEUS.

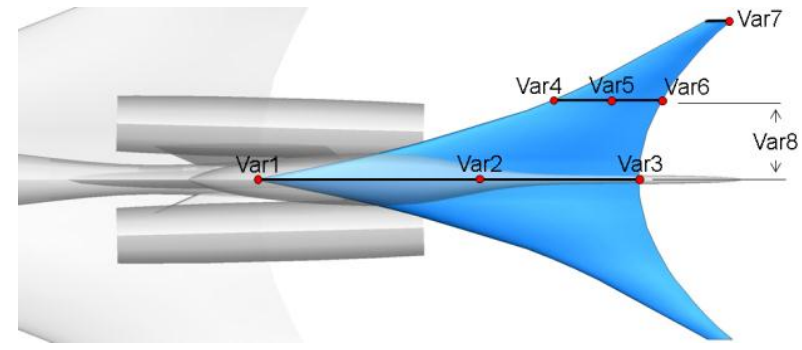

Figure 8. Horizontal tail parametric scheme for lift tailoring with PROTEUS.

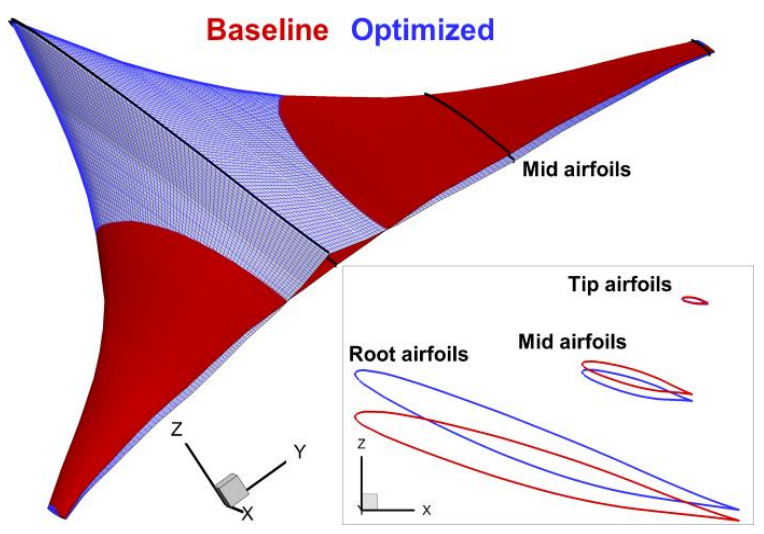

Figure 10. Comparison of baseline and optimized horizontal tail geometries for lift tailoring with PROTEUS.

\section{Conceptual Design and Integration of Supersonic Inlet with SUPIN and SUPIN2HRM}

The previous sonic boom optimization cases were conducted with flow-through nacelles (see Fig. 7) to avoid the additional computational expense associated with powered engine CFD analysis. A three-stage external compression supersonic inlet was generated with SUPIN by Karl Geiselhart from NASA Langley Research Center as shown in Fig. 11 for the final configuration of the previous lift tailoring optimization and at the same flight conditions. The supersonic inlet was integrated into the configuration using SUPIN2HRM as shown in Fig. 12. The power boundary conditions obtained from SUPIN at the fan face (shaded dark blue) and nozzle plenum (shaded red) are used to define the boundary conditions in CART3D for CFD analysis. The axial velocity contours for the CART3D CFD solution with powered engine boundary conditions is provided in Fig. 13. This figure also shows the initial shock of the SUPIN supersonic inlet impinged inside the cowl lip as is desired to reduce the amount of spillage from the engine. Figure 14 provides a comparison of the ground signatures for the original configuration with flow-through nacelles, and the configuration with a three-stage external compression supersonic inlet and powered boundary conditions. Here, the effect of powered engine boundary conditions on the ground signature propagated with SBOOM is small despite an increase in loudness of 2.5 PLdB. This is not always true and in some cases the configuration may require further work to recover a low-boom design. 


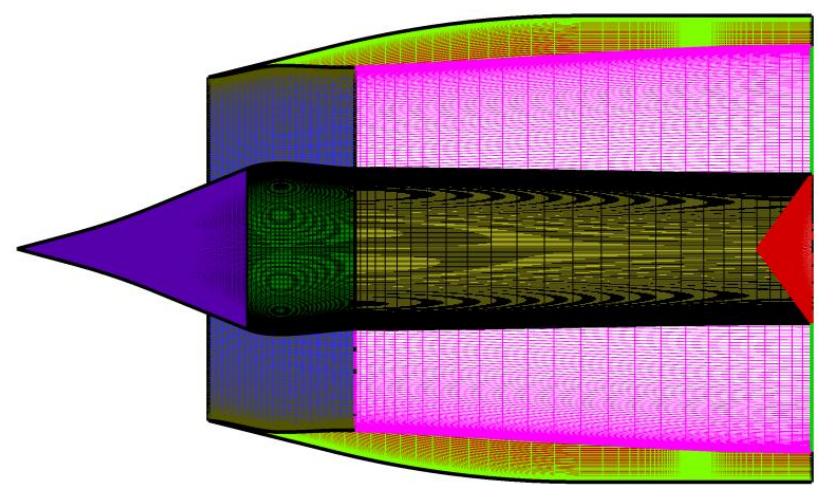

Figure 11. Three-stage external compression supersonic inlet design with SUPIN.

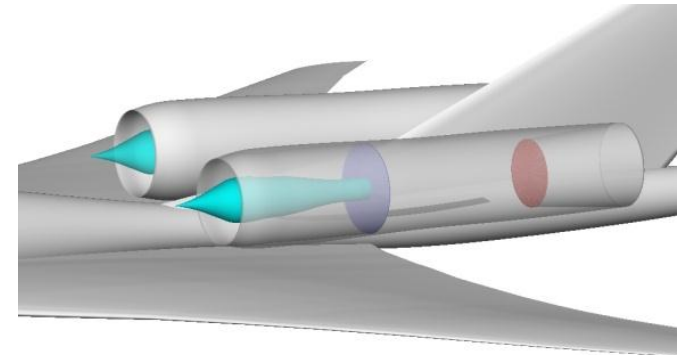

Figure 12. Three-stage external compression supersonic inlet integrated with SUPIN2HRM.

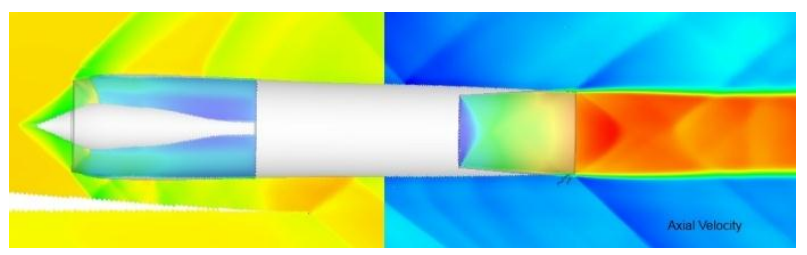

Figure 13. Axial velocity contours from CART3D CFD analysis of three-stage external compression supersonic inlet.

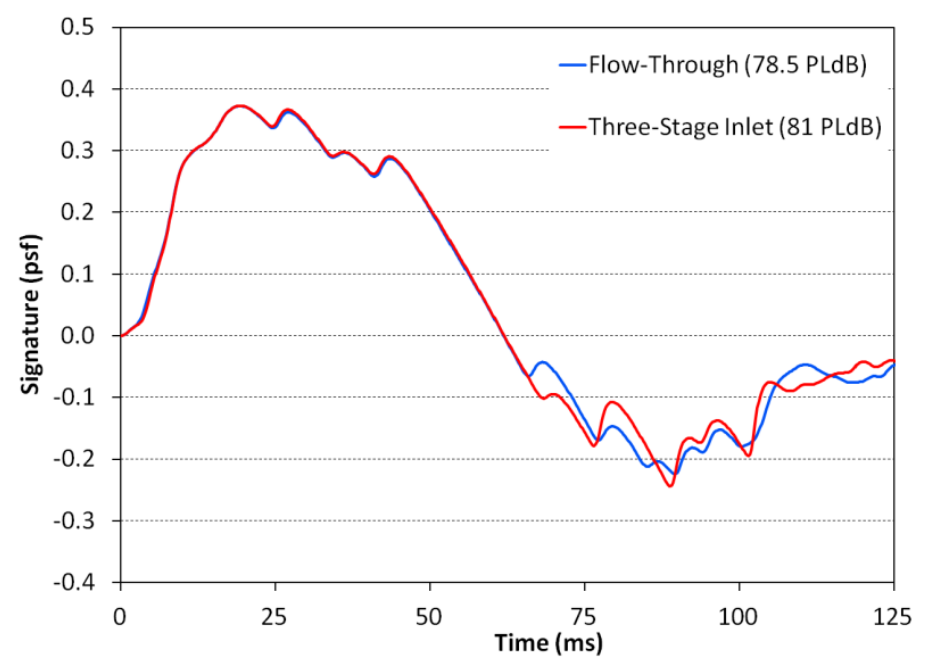

Figure 14. Ground signature comparison for configuration with flow-through and three-stage supersonic inlet design with SUPIN.

\section{Summary}

An overview of the current geometry modeling for the automated process of analysis and design of low-boom supersonic concepts starting with a VSP geometry has been provided. Several capability-enhancing design tools for VSP and practical case studies using these tools have been presented. Tools such as AST, BOSS, PROTEUS, and SUPIN2HRM are used to close the modeling gap that exists between conceptual geometry modeling tools like VSP and practical design applications. Geometry modeling gaps can vary depending on the application but the presented 
case studies also underscore the flexibility of using VSP with external geometry tools to meet specific design requirements during conceptual design.

AST was developed to easily manage the degrees of freedom for smooth geometry deformation for engineering definitions such as leading edge sweep, chord, and twist distributions. The number of design variables can be easily varied by changing the number of shape functions which define the geometry. BOSS allows volume-based shaping of the fuselage and pods with a high number (e.g., 120) of design variables for sonic boom minimization. PROTEUS is a flexible and rapid geometry parameterization tool which can deform lifting and non-lifting components based on engineering definitions such as camber, twist, and thickness distributions. More importantly, PROTEUS extends the capabilities of VSP by allowing detailed airfoil shape deformation which is critical to the lift-tailoring process needed for sonic boom minimization. Lastly, SUPIN2HRM allows the automated integration of supersonic inlets generated by the analysis-based design tool SUPIN into a VSP component-based point definition geometry, which can then be converted back to a VSP parametric model using the GEO2VSP tool.

\section{References}

${ }^{1}$ McDonald, R., “Open VSP,” www.openvsp.org [cited June 2012].

${ }^{2}$ Aftosmis, M., "Cart3D Resource Website,” http://people.nas.nasa.gov/ aftosmis/cart3d/cart3Dhome.html [cited June 2012].

${ }^{3}$ Ordaz, I., "Conversion of Component-Based Point Definition to VSP Model and Higher-Order Meshing," AIAA-2011-358, January 2011.

${ }^{4} \mathrm{Li}$, W., Shields, E., and Le, D., "Interactive Inverse Design Optimization of Fuselage Shape for Low-Boom Supersonic Concepts," AIAA-2008-136, January 2008.

${ }^{5} \mathrm{Li}, \mathrm{W} .$, "Rapid Parameterization Schemes for Aircraft Shape Optimization," AIAA-2012-964, January 2012.

${ }^{6}$ Ordaz, I., Rallabhandi, S., "Boom Minimization Framework for Supersonic Aircraft Using CFD Analysis," AIAA-20101506, January 2010.

${ }^{7}$ Kulfan, B. M. and Bussoletti, J. E., "Fundamental Parametric Geometry Representations for Aircraft Component Shapes," AIAA-2006-6948, September 2006.

${ }^{8}$ Slater, J., "Design and Analysis Tool for External-Compression Supersonic Inlets," AIAA-2012-16, January 2012.

${ }^{9}$ Wallace, H. D., Haefeli, R. C., and Kulsrud, H. E., "Sonic Boom Propagation in a Stratified Atmosphere with Computer Program," NASA CR-1299, April 1969.

${ }^{10} \mathrm{Li}$, W. and Rallabhandi, S. K., "Inverse Design of Low-Boom Supersonic Concepts Using Reversed Equivalent Area Targets," AIAA-2011-3498, June 2011.

${ }^{11}$ Rallabhandi, S. K., “Advanced Sonic Boom Prediction Using Augmented Burger's Equation,” AIAA-2011-1278, January 2011.

${ }^{12}$ Ordaz, I. and Li, W., "Adaptive Aft Signature Shaping of a Low-Boom Supersonic Aircraft Using Off-Body Pressures," AIAA-2012-0020, January 2012. 